\title{
Gastric microbiota and Helicobacter pylori in Indonesian population
}

\author{
Muhammad Miftahussurur ${ }^{1,2}$ (D) | Langgeng Agung Waskito ${ }^{2,3}$ (D) $\mid$ Hashem B El-Serag $^{4}$ | \\ Nadim J. Ajami ${ }^{4}$ | Iswan Abbas Nusi ${ }^{1}$ | Ari Fahrial Syam ${ }^{5}$ | Takashi Matsumoto ${ }^{3}$ | \\ Yudith Annisa Ayu Rezkitha ${ }^{6}$ | Dalla Doohan ${ }^{2,3}$ | Kartika Afrida Fauzia ${ }^{2,3}$ | \\ Ummi Maimunah $^{1}$ | Titong Sugihartono ${ }^{1}$ | Tomohisa Uchida ${ }^{7}$ | Yoshio Yamaoka ${ }^{1,3,4,8}$
}

\author{
${ }^{1}$ Division of Gastroentero-Hepatology, Department of Internal Medicine, Faculty of Medicine, Universitas Airlangga, Surabaya, Indonesia \\ ${ }^{2}$ Institute of Tropical Diseases, Universitas Airlangga, Surabaya, Indonesia \\ ${ }^{3}$ Department of Environmental and Preventive Medicine, Oita University, Faculty of Medicine, Yufu, Japan \\ ${ }^{4}$ Gastroenterology and Hepatology Section, Department of Medicine, Baylor College of Medicine, Houston, TX, USA \\ ${ }^{5}$ Division of Gastroenterology, Department of Internal Medicine, Faculty of Medicine, University of Indonesia, Jakarta, Indonesia \\ ${ }^{6}$ Faculty of Medicine, University of Muhammadiyah Surabaya, Surabaya, Indonesia \\ ${ }^{7}$ Department of Molecular Pathology, Faculty of Medicine, Oita University, Oita, Japan \\ ${ }^{8}$ Global Oita Medical Advanced Research Center for Health, Yufu, Japan
}

\section{Correspondence}

Yoshio Yamaoka, Department of

Environmental and Preventive Medicine,

Oita University Faculty of Medicine, 1-1

Idaigaoka, Hasama-machi, Yufu-City, Oita

879-5593, Japan.

Email:yyamaoka@oita-u.ac.jp

Muhammad Miftahussurur, GastroenteroHepatology Division, Department of Internal Medicine, Faculty of Medicine-Dr. Soetomo Teaching Hospital, Universitas Airlangga, Surabaya, Jalan Mayjend Prof. Dr. Moestopo No. 6-8 Surabaya 60131, Indonesia.

Email: muhammad-m@fk.unair.ac.id

\section{Funding information}

This study was funded by grants from the National Institutes of Health (DK62813) and the Grants-in-Aid for Scientific Research from the Ministry of Education, Culture, Sports, Science, and Technology (MEXT) of Japan (15H02657 and 221S0002 $16 \mathrm{H} 05191,16 \mathrm{H} 06279,18 \mathrm{KK} 0266$ and 19H03473) (YY). It was also supported by the Japan Society for the Promotion of Science Institutional Program for Young Researcher Overseas Visits (YY) and the Strategic Funds for the Promotion of Science and Technology from Japan Science and Technology Agency (JST) (YY). It was also supported by Riset Mandat Grant from Universitas Airlangga (340/

\begin{abstract}
Background: The profile of gastric mucosal microbiota has not yet been described in the Indonesian population where the prevalence of Helicobacter pylori is low.

Methods: This is a cross-sectional study analyzing 16S rRNA of 137 gastric biopsy specimens. We analyzed the association between gastric microbiota, H. pylori infection, and gastric mucosal damage.

Result: Among 137 analyzed samples, 27 were $H$. pylori-positive and 110 were $H$. pylori -negative based on culture, histology, and 16S rRNA gene analysis. Significantly lower $\alpha$-diversity parameters, including Pielou's index, was observed in $\mathrm{H}$. pylori-infected individuals compared with noninfected individuals (all $P<.001$ ). Among $H$. pylori-negative individuals, the permutational analysis of variance of Bray-Curtis dissimilarity distances showed a significant association with different ethnicities, suggesting some ethnic groups had specific microbiota profiles based on the presence of different operational taxonomic units. The linear discriminant analysis effect size (LEfSe) of the $\mathrm{H}$. pylori-negative group showed significant associations between the presence of Micrococcus luteus and Sphingomonas yabuuchiae with Timor and Papuan ethnicities, respectively. The presence of Bulledia sp and Atopobium sp was associated with the Javanese ethnicity. We observed lower $\alpha$-diversity scores in individuals with gastric mucosal damage and profiles with high abundances of Paludibacter sp and Dialister sp based on LEfSe analysis.
\end{abstract}


UN3.14/LT/2019). All authors have read and approved the final version of the manuscript.
Conclusion: Our findings suggest the presence of $H$. pylori is more correlated with a distinct microbiome profile than ethnic precedence.

KEYWORDS

Helicobacter pylori, Indonesia, microbiome

\section{1 | INTRODUCTION}

The gastric mucosal environment was thought to be sterile due to the presence of gastric acid until the discovery of Helicobacter pyIori, ${ }^{1}$ a causal agent for gastritis, peptic ulcer diseases, and gastric cancer. ${ }^{2}$ The use of $16 \mathrm{~S}$ rRNA next-generation sequencing revealed trillions of gastric mucosal microbiota, most of which cannot be cultivated. ${ }^{3}$ Further, there are possibly different microbial communities between stomachs that are healthy and those with diseases (eg, gastric cancer). ${ }^{4,5}$ The presence of $H$. pylori greatly influences microbial dysbiosis $^{6,7}$ and the risk of gastric cancer. ${ }^{6}$

The composition of the gastric microbiota is influenced by age, ${ }^{8}$ diet, ${ }^{9}$ and ethnicity. ${ }^{10}$ Ethnicity has been proposed to influence the gastric microbiome through diet patterns and possibly genetic factors. ${ }^{4}$ A study conducted in China reported a significant difference in the richness and diversity of the gut microbiome among three ethnic groups (Yao, Zhuang, and Han), ${ }^{11}$ and similar findings were reported in Europe and the United State, indicating that ethnicity contributed to the intergroup dissimilarity of gut microbiota composition. ${ }^{12,13}$ However, most of these studies identified gut microbiota using stools samples. ${ }^{13}$ Few studies have evaluated the gastric microbiome using gastric mucosa samples, which provide a more accurate representation of the resident gastric microbiome compared with the transient one. ${ }^{1415}$

Indonesia is a country with several hundred ethnicities and a low prevalence of $H$. pylori infection. ${ }^{16}$ The Javanese ethnicity is the largest in Indonesia, contributing $~ 40 \%$ of the population, most of whom live on Java Island. Several other ethnic groups live on other islands, such as the Kupang, Timorese, and Papuan ethnicities in eastern areas of Indonesia and the Nias and Batak tribes in the western tip of Indonesia. These ethnic groups have distinctive cultures and diets that could influence the composition of gut microbiota. The lower prevalence of $\mathrm{H}$. pylori allows us to examine the role of other organisms in the pathogenesis of gastric disorders. Therefore, we investigated the composition of gastric mucosal microbiota in an Indonesian population and analyzed the associations between microbiome composition, ethnicity, and gastric disease status.

\section{2 | METHODS}

\section{1 | Patients and samples}

From January 2014 to August 2016, we collected 784 gastric mucosal specimens from 784 individuals in 11 cities across Indonesia, including sites located on the five largest islands (Figure S1). We excluded patients with a history of $H$. pylori eradication therapy, partial or total gastrectomy, or a contraindication to upper endoscopy with biopsies. Gastric mucosal specimens for microbiome analysis were obtained from the lesser curvature of the antrum, $\sim 3 \mathrm{~cm}$ from the pyloric ring. They were preserved in transport medium and homogenized in $500 \mu \mathrm{L}$ of phosphate-buffered saline for $\mathrm{H}$. pylori culture. The remaining homogenized specimens were stored into $-80^{\circ} \mathrm{C}$ and used for DNA extraction. We took two additional biopsy specimens from the antrum and corpus for histologic examination. All specimens were obtained using Radial Jaw 4 forceps (Boston Scientific).

We excluded 97 patients who did not have a complete histology result. The remaining 687 samples comprised patients who were Javanese (203 patients), Bataknese (113 patients), Bugis (87 patients), Chinese Indonesian (100 patients), Balinese (60 patients), Dayak (47 patients), Papuan (47 patients), and Timor (30 patients). We randomly selected 192 samples with the selection algorithm weighted for each ethnic group and the location regardless of $\mathrm{H}$. pylori status. These samples included patients who were Javanese (48 samples); Batak (30 samples); Bugis (35 samples); Chinese Indonesian (17 samples); Balinese (19 samples); Dayak (14 samples); Papuan (13 samples); and Timor (16 samples).

\subsection{DNA extraction and PCR amplification of $16 \mathrm{~s}$ rRNA sequences}

DNA was extracted using the DNeasy Blood \& Tissue Kit (QIAGEN) and was concentrated using a DNA Clean \& Concentrator (Zymo Research). Extracted DNA was stored at $-20^{\circ} \mathrm{C}$.

We followed the manufacturer's protocol for preparing the $16 \mathrm{~S}$ rRNA gene DNA library (Illumina Inc, San Diego, CA). The V3-V4 region of the bacterial 16S rRNA gene was amplified using the universal primers 341F (5'-TCGTCGGCAGCGTCAG ATGTGTATAAGAGACAGCCTACGGGNGGCWGCAG-3') and 805R (5'-GTCTCGTGGGCTCGGAGATGTGTATAAGAGACAG GACTACHVGGGTATCTAATCC3-3'). ${ }^{17}$ PCR amplification was performed using KAPA HiFi HotStart Ready Mix (KAPA Biosystems Inc) with denaturing at $95^{\circ} \mathrm{C}$ for 3 minutes; 35 cycles of amplification at $95^{\circ} \mathrm{C}$ for 30 seconds, $55^{\circ} \mathrm{C}$ for 30 seconds, and $72^{\circ} \mathrm{C}$ for 30 seconds; and final elongation at $72^{\circ} \mathrm{C}$ for 5 minutes. The Nextera XT Index kit (Illumina Inc) was added, and samples underwent an additional eight cycles of PCR. Amplicons were purified using Agencourt AMPure XP magnetic beads (Beckman Coulter). We validated the DNA library using a bioanalyzer MCE-202 MultiNA system (Shimadzu) and QuantiFluor dsDNA system (Promega Corporation). Finally, 
the pooled 5 pM DNA library was denatured with $0.2 \mathrm{~N} \mathrm{NaOH}$ and mixed with PhiX Control v3 (Illumina Inc) to $15 \%$ of the final concentration as described in Illumina's procedure. Paired-end sequencing was performed using the next-generation sequencer MiSeq platform (Illumina Inc) with the MiSeq Reagent Kit version 3 (2 $\times 300$ bp Paired-End Reads; Illumina Inc).

\section{3 | Sequence data analysis}

We removed index and adapter sequences and low-quality reads using Trimmomatic. ${ }^{18}$ The read-retention parameter was having a Phred quality score $\geq 20$. The high-quality reads underwent close reference operational taxonomic unit (OTU) picking using vsearch. ${ }^{19}$ The reference database was Greengenes version 13.5. ${ }^{20}$ Using a $99 \%$ identity parameters, we generated an OTU table and the representative sequences of each OTUs. These sequences were aligned using MAFFT, ${ }^{21,22}$ and a phylogenetic tree was constructed using FastTree. ${ }^{23}$ We imported the OTU table, representative sequences, and phylogenetic tree as artifacts to the QIIME 2 analysis platform (https://qiime2.org). The $\alpha$-diversity analysis, including species richness, Pielou's evenness index, ${ }^{24}$ Faith's phylogenetic diversity index, ${ }^{25}$ and Shannon's diversity index, ${ }^{26}$ was analyzed using QIIME2. The $\beta$-diversity analysis using Bray-Curtis dissimilarity matrix ${ }^{27}$ was also performed using QIIME 2. The Bray-Curtis dissimilarity matrix was used to draw principal coordinate analysis figures in R. The permutational analysis of variance (PERMANOVA) with Bonferroni correction was calculated using the Adonis function from the vegan package in $\mathrm{R}^{28}$

We performed linear discriminant analysis effect size (LEfSe) to identify OTUs that are associated with that are likely to explain differences between groups, specifically those based on ethnicity and disease status. ${ }^{29}$ In addition, we also performed analysis of variance (ANOVA) to identify differences in relative abundance of specific OTU between ethnic groups and diseases. The LEfSe and ANOVA analysis were performed using the Calypso web-based analysis platform version $8.84,{ }^{30}$ available at (http://cgenome.net:8080/calypso8.84/faces/uploadFiles.xhtml).

\subsection{Determination of $H$. pylori infection}

We determined the $H$. pylori infection status based on $H$. pylori culture and histologic examination.

\subsection{1 | Culture}

Antral biopsy specimens were homogenized, inoculated on an H. pylori -selective agar plate, and incubated up to 10 days in microaerophilic condition $\left(10 \% \mathrm{O}_{2}, 5 \% \mathrm{CO}_{2}\right.$, and $\left.85 \% \mathrm{~N}_{2}\right)$ at $37^{\circ} \mathrm{C}$. Subsequently, colonies were sub-cultured in antibiotic-free MuellerHinton II agar medium (Becton Dickinson) supplemented with $10 \%$
Horse Blood and incubated in the same microaerophilic conditions. H. pylori stock was stored in Brucella Broth (Difco) with 10\% dimethyl sulfoxide and $10 \%$ horse serum at $-80^{\circ} \mathrm{C}$.

\subsubsection{Histologic analysis}

All biopsy material for histologic testing was fixed in $10 \%$ buffered formalin and embedded in paraffin. Serial sections were stained with hematoxylin and eosin and May-Giemsa stain. The degree of neutrophil activity, inflammation, atrophy, intestinal metaplasia, and $H$. pylori bacterial density was classified into four grades based on the updated Sydney system (0, normal; 1, mild; 2, moderate; and 3, marked). ${ }^{31}$ Samples with bacterial loads of grade 1 or higher were considered positive for $\mathrm{H}$. pylori. We defined gastric mucosal damage, that is, an observation of both neutrophil or monocyte infiltration, as gastritis. Precancerous lesions were defined as an observation of either atrophic gastritis or intestinal metaplasia. To increase the accuracy of $H$. pylori detection, we performed immunohistochemistry of all samples, as described previously. ${ }^{32}$ The experienced pathologist (TU) who evaluated the specimens in this study also performed studies for Myanmar, Vietnam, Bhutan, the Dominican Republic, and Indonesia. ${ }^{33-38}$

\section{3 | RESULTS}

Among the gastric mucosal samples from 192 patients, 14 samples produced suboptimal $16 \mathrm{~S}$ rRNA gene libraries for sequencing. Therefore, 178 samples were sequenced for microbiome profiling targeted at the V3-V4 regions of the 16S rRNA gene. We found $16.3 \%(29 / 178)$ of specimens were H. pylori -positive based on histology and confirmed by immunohistochemistry or culture.

We performed OTU selection and identified 68 classes, 135 orders, and 253 families of bacteria. We excluded samples that had conflicting $H$. pylori status results between histology/culture and 16srRNA. Among excluded samples, 39 were designated $H$. pylori-negative based on histology/culture but had relative abundances of $H$. pylori greater than $2 \%$ (range $2.02 \%-83.12 \%$ ) and 2 were $H$. pylori-positive based on histology/culture but had low relative abundances of H. pylori ( $0.67 \%$ and $0.46 \%)$.

A total of 137 samples were included in the final analysis; 27 were $H$. pylori-positive and 110 were $H$. pylori-negative based on histologic examination, culture, and $16 \mathrm{~S}$ rRNA gene sequencing. The mean age of this group was $48.34 \pm 14.8$ years, and 79 samples were from male patients and $58(42.3 \%)$ from female patients. $H$. pylori-positive patients were significantly older than H. pylori-negative ones (52.2 vs 46.5 years, respectively; $P=.032$ ). There were $22(16.7 \%)$ samples from patients with gastritis; 20 (90.9\%) were H. pylori-negative, and 2 (9.1\%) were H. pylori-positive. Further, 56 (40.9\%) samples were from patients with atrophic gastritis or intestinal metaplasia; 31 (55.4\%) were H. pylori-negative, and 25 (44.6\%) were H. pylori-positive. 


\subsection{Gastric microbiota diversity and $H$. pylori positivity}

We observed significantly lower species richness, Pielou's evenness, Shannon's diversity index, and Faith's phylogenetic diversity in $H$. pylori-positive samples $(n=22)$ compared with $H$. pylorinegative samples $(\mathrm{n}=110$ ) (all $P<.001$, Figure $1 \mathrm{~A}$ ). The $H$. pyloripositive group had a lower number of observed OTUs, suggesting lower diversity in $\mathrm{H}$. pylori-positive samples compared with $\mathrm{H}$. pylori-negative samples. The H. pylori-positive group also had a lower Pielou's index score for evenness, suggesting dominance by one or more OTUs. We observed higher OTU similarity in the $\mathrm{H}$. pyloripositive than the $H$. pylori-negative group as indicated by Faith's phylogenetic diversity. Shannon's diversity index also indicated that the H. pylori-positive group was less diverse than the H. pylorinegative one. There was a significant negative correlation between Shannon's diversity index and age $(r=-0.31, P<.001$, Figure 1B).
Similarly, the H. pylori-negative group showed a significant negative correlation between Shannon's diversity index and age $(r=-0.25$, $P=.0089 ;$ Figure S2).

Next, we evaluated differences in $\beta$-diversity between $H$. pylori-negative and $H$. pylori-positive groups using the Bray-Curtis dissimilarity matrix. $H$. pylori-positive and $H$. pylori-negative groups exhibited different clustering positions (Figure 2). PERMANOVA analysis of the Bray-Curtis distance matrix supports our observation of distinct clustering of the $\mathrm{H}$. pylori-positive compared with the $H$. pylori-negative groups, highlighting their differences in microbial composition $\left(R^{2}=0.248, P<.001\right)$. The relative abundance of Family Helicobacteraceae (74.6\%) predominated in H. pylori-positive samples (Figure 3), whereas the Families Streptococcaceae (25.9\%), Prevotellaceae (13.5\%), and Veillonellaceae (10.8\%) predominated in the H. pylori-negative group. On the species level, H. pylori had $76.2 \%$ relative abundance in the $H$. pylori-positive group. In the $H$. pylori-negative group, Prevotella melaninogenica had $23.5 \%$ relative
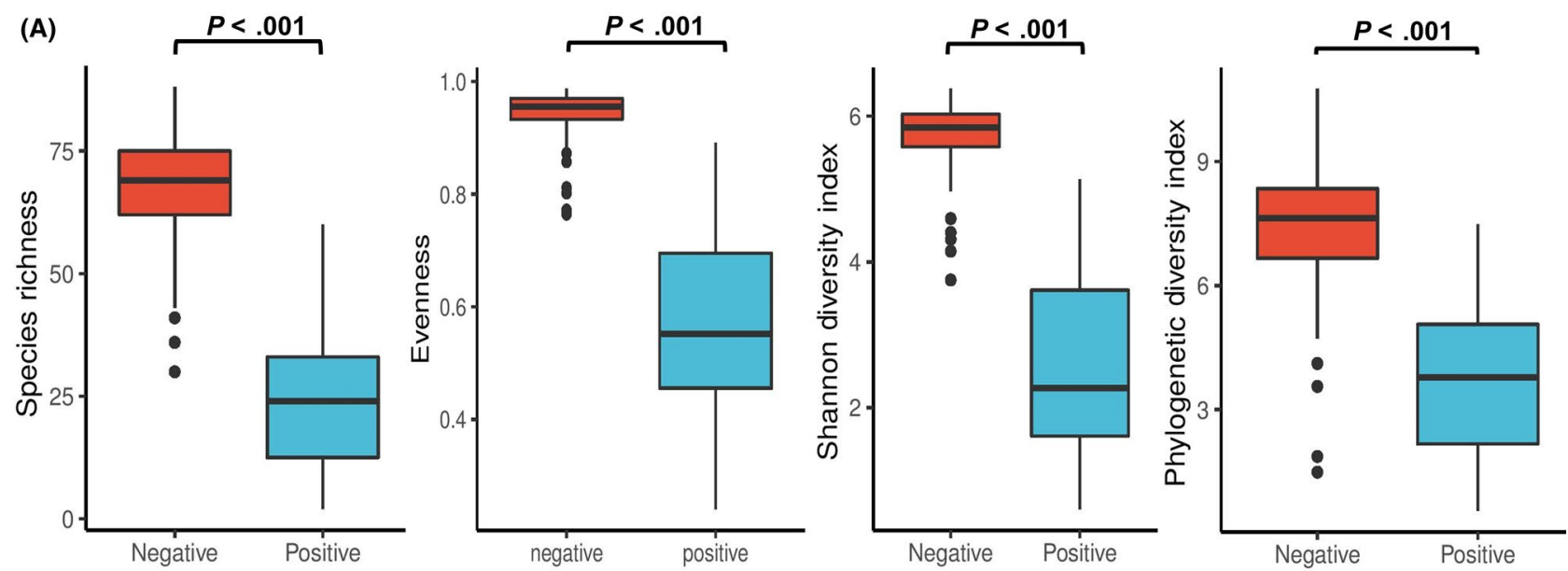

H. pylori status

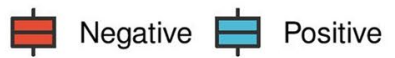

(B)

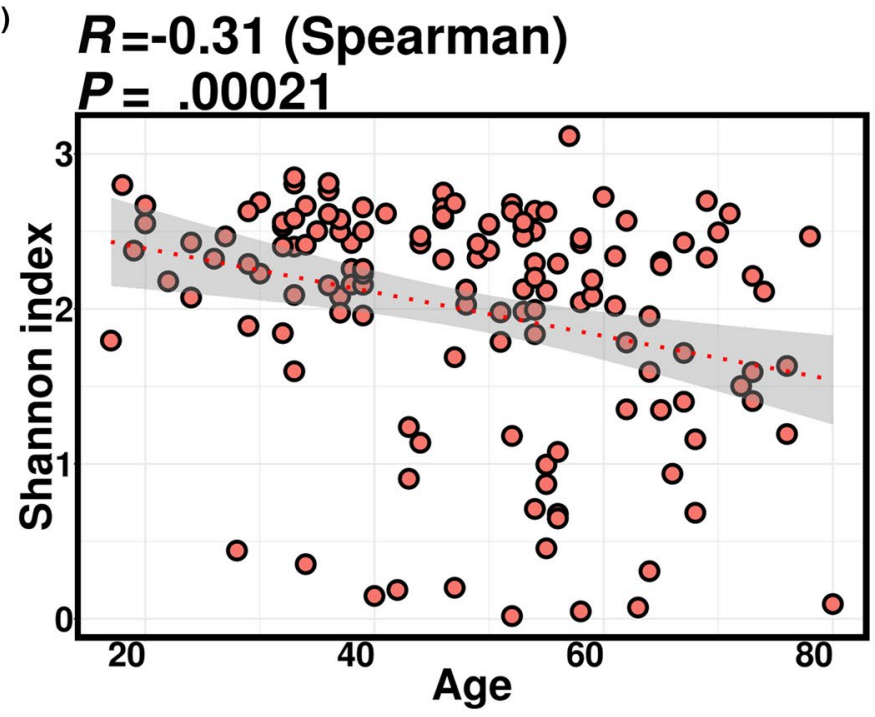

FIGURE 1 Association of $\alpha$-diversity with the Helicobacter pylori infection and age. A H. pylori infection significantly reduced $\alpha$-diversity parameters and Pielou's phylogenetic diversity. B. Shannon's diversity index was significantly reduced as age increased 
abundance, Rothia mucilaginosa had 19.5\%, Veillonella dispar had $18.6 \%$, and Haemophilus parainfluenzae had $7.2 \%$.

\subsection{Microbiome diversity and ethnic groups}

There were significant differences in species richness, evenness, Faith's phylogenetic diversity, and Shannon's diversity indexes (all

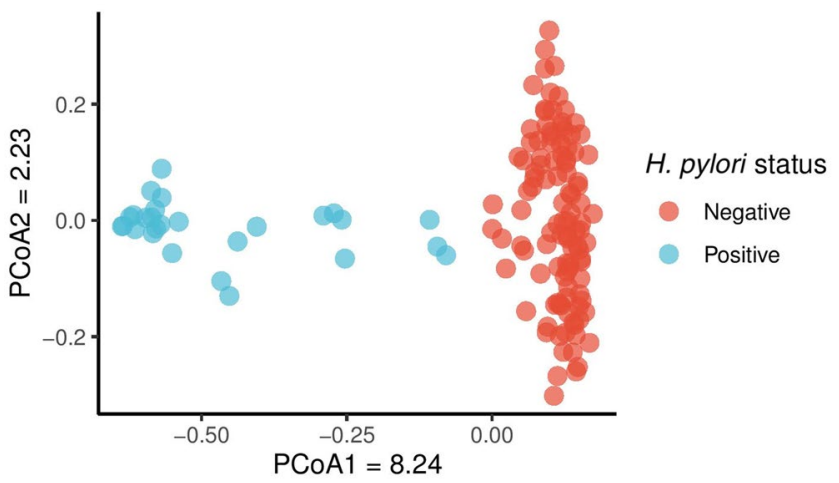

FIGURE 2 The $\beta$-diversity of 137 analyzed samples between Helicobacter pylori-positive and $\mathrm{H}$. pylori-negative subjects. $\mathrm{H}$. pylori-positive and $\mathrm{H}$. pylori-negative samples were clustered separately. PERMANOVA analysis showed a significant difference in $\beta$-diversity between $H$. pylori-positive and $H$. pylori-negative groups $(P<.001)$

(A)

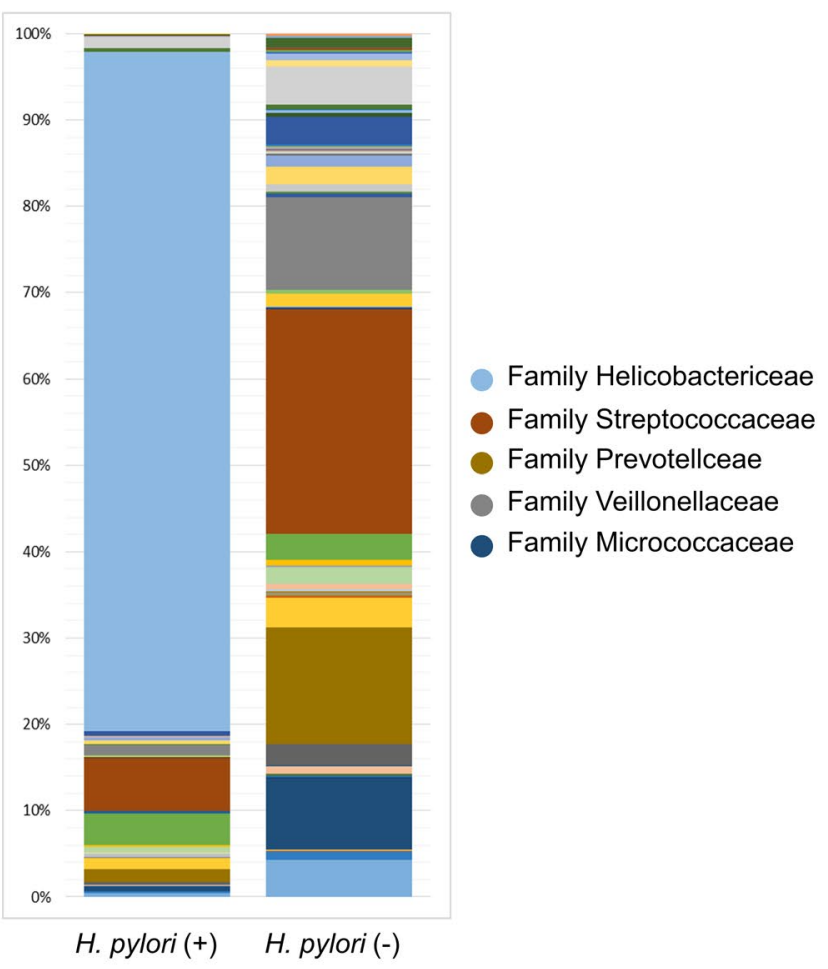

$P<.001)$ among the different ethnic groups (Figure 4A). Pairwise analysis showed the Timor $(n=9)$, Balinese $(n=7)$, and Papuan $(n=9)$ ethnic groups had significantly lower richness, evenness, Faith's phylogenetic diversity index, and Shannon's diversity index than Javanese $(n=30)$, Dayak $(n=12)$, Chinese $(n=11)$, Bugis $(n=33)$, and Bataknese ( $n=26$; Figure S3). Interestingly, in the H. pylori-negative group, there were no significant differences in richness, evenness, Faith's phylogenetic diversity index, or Shannon's diversity index $(P=.087, P=.065, P=.222$, and $P=.078$, respectively) among ethnic groups (Figure $4 \mathrm{~B}$ ), but the $\beta$-diversity was significantly associated with ethnic group based on the PERMANOVA analysis of BrayCurtis dissimilarity matrix $\left(P=.023, R^{2}=0.086\right)$. This result suggests a predominant OTU in some ethnic groups. We performed alpha and phylogenetic diversity analyses comparing $H$. pylori-positive and $H$. pylori-negative samples in each ethnic group and observed significantly lower species richness, Pielou's evenness, Shannon's diversity index, and Faith's phylogenetic diversity index in the positive group compared with the negative group (all $P<.001$; Figure S3).

To find candidate OTUs related to each ethnic group, we performed LEfSe analysis of only H. pylori-negative samples. We observed that Streptococcus sp and Micrococcus luteus were associated with the Timor ethnicity (linear discriminant analysis [LDA] score $=4.78$ and 3.56, respectively). Sphingomonas yabuuchiae was associated with the Papuan ethnicity (LDA score $=3.82$ ). Bulledia sp and Atopobium sp were associated with the Javanese ethnicity (LDA score $=3.89$ and 3.87 , respectively). Neisseria subflava, Leptotrichia sp, Prevotella nanciensis
(B)

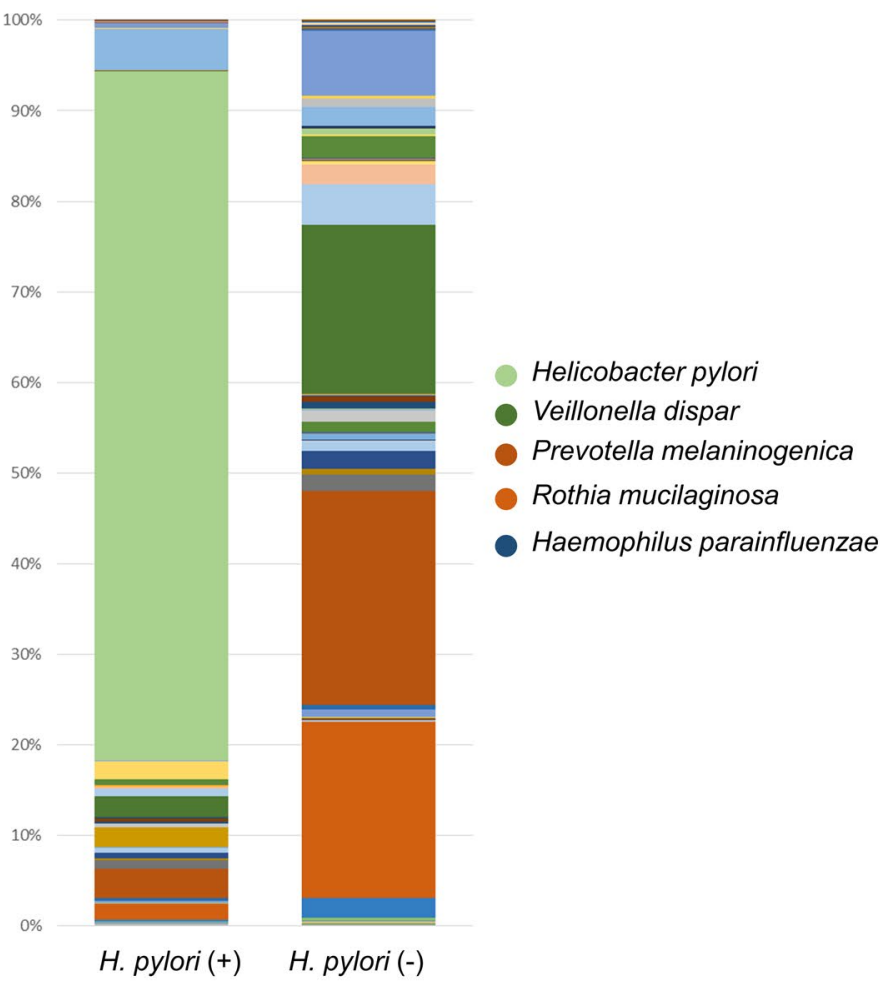

FIGURE 3 Relative abundance of gastric microbiota between Helicobacter pylori-positive and $H$. pylori-negative subjects. At the family level, we observed that Helicobacteraceae accounted for $74.6 \%$ of the relative abundance in the $H$. pylori-positive group. At the species level, H. pylori was the most dominant OTU, accounting for $76.2 \%$ of the relative abundance in the $H$. pylori-positive group 

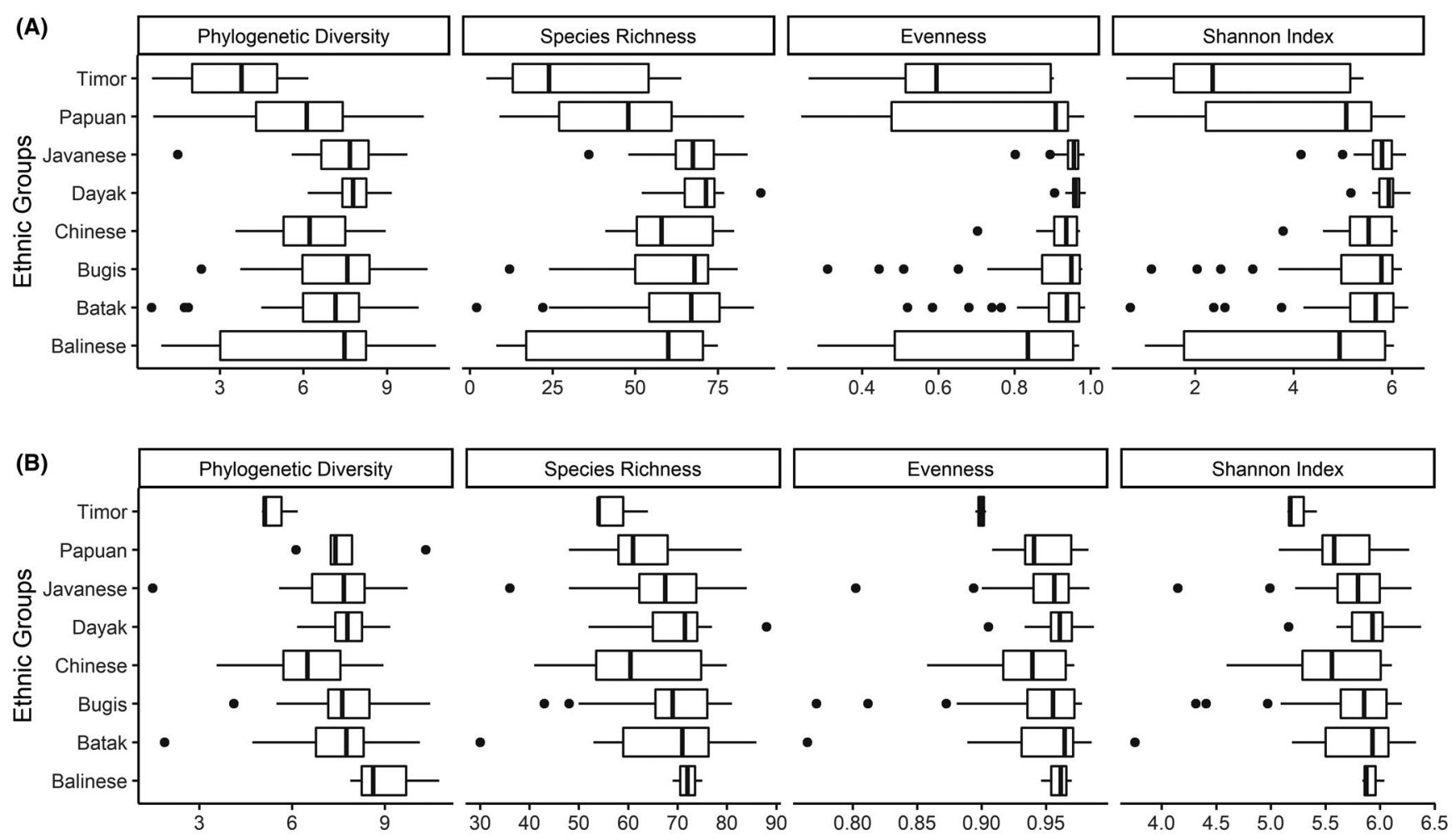

FIGURE 4 The $\alpha$-diversity among ethnic group in Indonesia. The $\alpha$-diversity among all Indonesian samples was significantly different, suggesting that ethnic group influences gastric microbial composition in Indonesia. When we analyzed only the Helicobacter pylori-negative samples, there were no observed differences among ethnic groups, suggesting that $H$. pylori infection is a stronger influence on microbial composition than ethnic group

(LDA score $=4.16,3.91$ and 3.77, respectively), and Actinobacillus parahaemolyticus (LDA score $=3.90$ ) were associated with the Dayak ethnicity. Schlegelella sp was associated with the Bataknese ethnicity (LDA score $=3.83$ ). Capnocytophaga sp, Prevotella intermedia, and Mycoplasma sp were associated with the Balinese ethnicity (LDA score $=3.94,3.94$ and 3.48, respectively). ANOVA analysis showed significant differences in the relative abundances of Bulledia sp, Prevotella nanciensis, and Prevotella intermedia among the ethnic groups $(P=.022$, $P=.048$ and $P<.001$, respectively; Figure S4).

\section{3 | The microbiome diversity and gastroduodenal diseases}

Analysis of all included samples showed significant lower Pielou's phylogenetic diversity index and $\alpha$-diversity parameters, including Shannon's diversity index, OTU evenness, and species richness, among individuals with gastric mucosal damages (either gastritis ( $n=22$ ) or precancerous lesions $(n=56)$ group) than those with normal mucosa $(n=59$ ) (all $P<.001$ ). When only analyzing the $H$. pylori-negative group, there was no significant difference in species richness between the gastritis $(n=20)$ group and the normal group $(n=59 ; P=.16)$. However, the gastritis group had significantly lower OTU evenness and Shannon's diversity index $(P=.02$ and $P=.046$, respectively).
The LEfSe analysis showed H. pylori and Helicobacter $\mathrm{sp}$ OTUs are likely to be factors in gastric mucosal damage (LDA score $=4.83$ and 3.69, respectively; Figure 5A). ANOVA analysis confirmed a higher abundance of both $H$. pylori and Helicobacter $\mathrm{sp}$ in the gastritis group versus the normal group $(P<.001$ and $P=.012$, respectively). In the H. pylori-negative group, Lactobacillus sp, Paludibacter sp, Dialister sp, and Scardovia sp are likely to be factors in gastric mucosal damage (LDA score $=3.86,3.56,3.52$ and 3.52, respectively; Figure 5B). Furthermore, ANOVA analysis showed significantly higher relative abundances of Lactobacillus $\mathrm{sp}$, Paludibacter sp, Dialister sp, and Scardovia sp in the gastritis group versus the normal group $(P=.03, P=.025, P=.03$, and $P=.019$, respectively).

There were significant differences in species richness, OTU evenness, and Shannon's diversity index observed between the precancerous (ie, atrophic gastritis, intestinal metaplasia), gastritis, and normal groups (all $P<.001$ ). The lowest diversity was observed in the precancerous group, followed by the gastritis and normal groups. Among H. pylori-positive samples, the species richness, OTU evenness, and Shannon's diversity index were significantly lower in the precancerous group compared with the gastritis group $(P=.02, P=.023$, and $P=.012$, respectively). In the $H$. pylori-negative group, the evenness was reduced in the gastritis and precancerous groups compared with the normal group $(P=.067)$, whereas there were no significant differences 
(A)

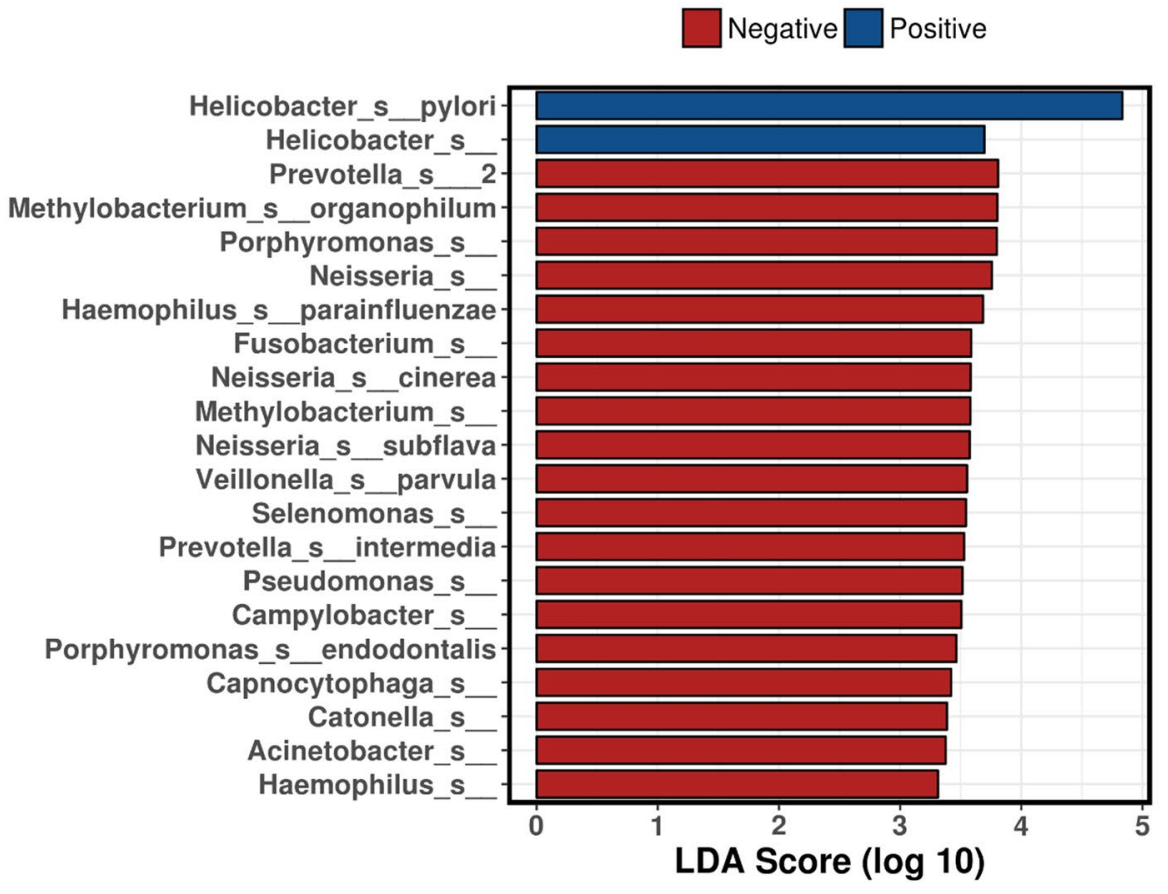

(B)

Cancer.Precusor $\square$ Gastritis $\square$ normal

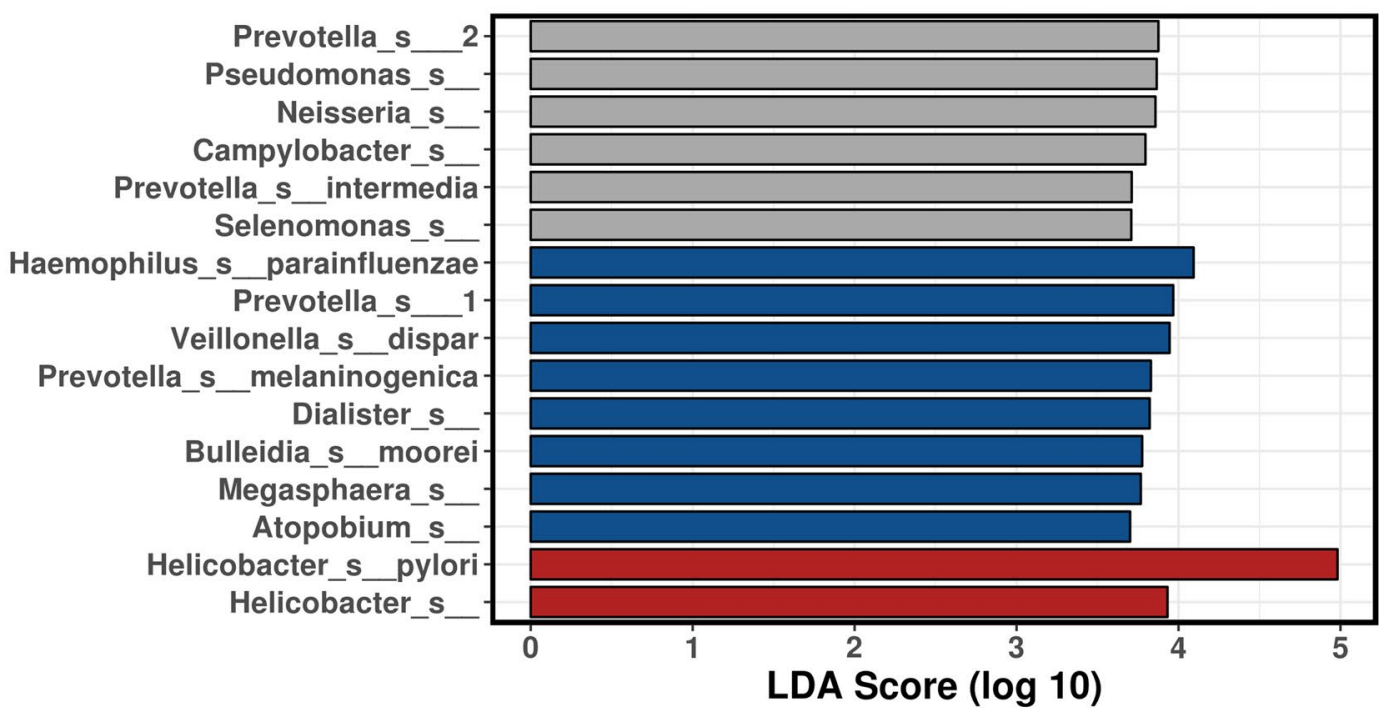

FIGURE 5 The LEfSe analysis of gastric abnormality among Indonesian patients. A. Among all analyzed data, Helicobacter pylori and other Helicobacter sp showed the most associated to the gastric abnormalities, regardless the type of the abnormality. B. When classified into three groups, the H. pylori and other Helicobacter sp. showed the most associated to the gastric cancer precursor group (either atrophic gastritis or intestinal metaplasia)

in Shannon's diversity index and species richness. LEfSe analysis showed Corynebacterium sp and Rothia dentocariosa are likely to be factors in the precancerous group (LDA score $=3.56$ and 3.47 , respectively), and Paludibacter $\mathrm{sp}$ is likely to be a factor in with the gastritis group (LDA score $=3.43$; Figure 6). ANOVA analysis showed Rothia dentocariosa had significantly higher relative abundance in the precancerous group compared with the other groups $(P=.037)$.

\section{DISCUSSION}

This is the first study to analyze how gastric mucosal microbiota is related to $\mathrm{H}$. pylori infection and ethnicity in Indonesia. Similar to previous studies, ${ }^{39,40}$ we showed that $H$. pylori infection status was an important factor in determining the gastric microbiota diversity in Indonesia, with lower $\alpha$-diversity in the positive group compared with the negative group. In addition to $H$. pylori infection, increasing 


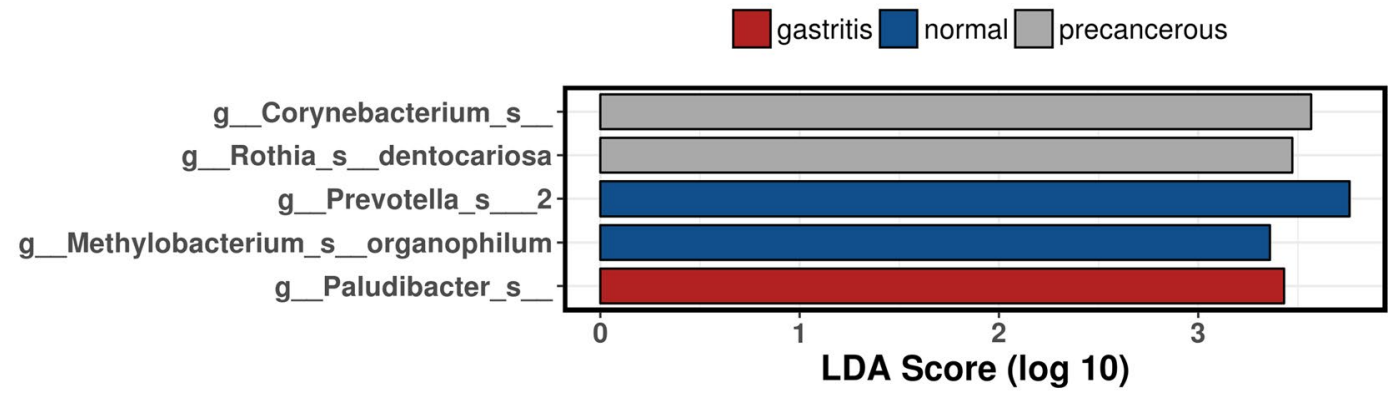

FIGURE 6 The LEfSe analysis of gastric abnormalities among the Helicobacter pylori-negative group

age was also significantly associated with a lower diversity index consistent with previous observations. ${ }^{41,42}$ As expected, $\beta$-diversity analysis showed family Helicobacteraceae had extremely high relative abundance in $\mathrm{H}$. pylori-positive group, representing more than three-quarters of the total family output. ${ }^{39}$ Also consistent with previous studies, ${ }^{39,43}$ we found that Prevotella and Rothia were the two highest abundance bacterial families within the $H$. pylori-negative group in Indonesia.

In this study, we found and excluded from the analysis 39 cases with discordance in $\mathrm{H}$. pylori finding between histologic analysis and $16 s$ rRNA analysis which was excluded for further analysis. This discordance might due to very low bacterial load in the stomach which could not be observed by histologic analysis. Additionally, there are several cases that $H$. pylori colonized in the patchy state.

We found that the Timor, Balinese, and Papuan ethnic groups had significantly less microbiota richness and evenness compared with the Javanese, Dayak, Chinese, Bugis, and Bataknese ethnic groups. When these analyses were stratified by $H$. pylori status, there were no significant differences in gastric microbiota composition between the different ethnic groups among $H$. pylori-positive individuals, likely due to $\mathrm{H}$. pylori dominating the microbiota composition. Additionally, we found no significant differences in $\alpha$-diversity between the different ethnic groups among $H$. pylori-negative individuals. Therefore, these differences were more likely explained by the higher prevalence of $\mathrm{H}$. pylori infection in these ethnic groups as the prevalence had been shown in previous studies. ${ }^{16,44}$ However, among $H$. pylori-negative individuals, we found a significant association between $\beta$-diversity and ethnic groups based on PERMANOVA analysis, suggesting the presence of a predominant OTU in each ethnic group. Streptococcus sp was the predominant species in $\mathrm{H}$. pylori-negative subjects of Timor ethnicity, Sphingomonas yabuuchiae was predominant in those of Papuan ethnicity, and Bulledia sp was predominant in those of Javanese ethnicity.

The analysis of the microbiota factors that could explain the differences between healthy tissue and gastric mucosal damage showed that the H. pylori and Helicobacter sp OTUs had the largest effect size in with respect to gastric mucosal damage. However, Indonesia has a low prevalence of $H$. pylori infection but a high prevalence of dyspepsia cases in outpatient clinics. ${ }^{16,45,46}$ Therefore, we focused on $\mathrm{H}$. pylori-negative subjects when analyzing association between histologic gastric disease and gastric microbiota. While there was no significant difference in species richness between subjects with and without gastric mucosal damage, we found significant difference in OTU evenness, which could be caused by the predominance of specific bacteria in the stomachs of subjects with abnormalities. This finding was also supported by the significant differences observed in $\beta$-diversity analysis. We found that Lactobacillus $\mathrm{sp}$, Paludibacter $\mathrm{sp}$, and Dialister sp were significantly higher in subjects with gastric abnormalities. Additionally, LEfSe analysis showed that Paludibacter $\mathrm{sp}$ had the largest effect size in with respect to the gastritis group. Paludibacter is a genus of a Gram-negative bacteria that are nonmotile; strictly anaerobic; and lack oxidase, catalase, and nitrate reducing activity. ${ }^{47}$ One previous study reported that Paludibacter sp was significantly reduced in subjects receiving proton-pump inhibitor treatment. ${ }^{48}$ Therefore, there could be a possible role for these bacteria in the development of gastric disease. Dialister sp found in gastric mucosa was hypothesized to be a members of oral pathogenic taxa that could have a role in the development of gastric cancer. ${ }^{49}$ Dialister sp was previously reported to form co-occurring interactions that strengthen with gastric disease progression. ${ }^{49}$

The study was limited by the relatively small sample size for some of the ethnic groups. This small sample size resulted in small numbers in some groups. To mitigate this effect, we combined atrophic gastritis and intestinal metaplasia into the gastric cancer precursor group. Therefore, the associated microorganism needs to be interpreted with caution. The further study with bigger sample number is necessary for more clear association. In addition, we did not include transcriptome, proteomic, or metabolomic profiles; therefore, we cannot draw inferences regarding functional differences. The use of DNA analysis does not confirm whether microorganisms are alive or not. Finally, the findings reflect significant association that may not be interpreted as the causal. Future studies are needed to determine the correlations between gastric microbiota and the natural history of gastric disease and get a better understanding of the role of gastric microbiota.

\section{5 | CONCLUSIONS}

Our study confirmed that $H$. pylori infection overwhelms other gastric microbiota, dominating the gastric microbial composition. The effect of $H$. pylori infection on the gastric microbiomes of various ethnic groups is stronger than the effect of the ethnic group 
designation itself. Among H. pylori-negative individuals, Paludibacter $\mathrm{sp}$ and Dialister sp were the species that could explain gastric mucosal damage in Indonesia.

\section{ACKNOWLEDGEMENTS}

None.

\section{CONFLICT OF INTEREST}

The authors declare there was no potential competing interest.

\section{AUTHORS' CONTRIBUTIONS}

$Y Y, M M$, and AFS conceived and designed the experiments. MM, TM, TU, and LAW performed the experiments. MM, YY, LAW, HBE, and NJA analyzed the data. AFS, HBE, NJA, DD, PBS, YAAR, and IAN contributed reagents/materials/analysis tools. MM, LAW, YY, and HBE wrote the manuscript.

\section{ETHICS APPROVAL}

Written informed consent was obtained from all patients. The study protocol was approved by the Ethics Committee of Dr Cipto Mangunkusumo Teaching Hospital (Jakarta, Indonesia), Dr Soetomo Teaching Hospital (Surabaya, Indonesia), Dr Wahidin Sudirohusodo Teaching Hospital (Makassar, Indonesia), and Oita University Faculty of Medicine (Yufu, Japan).

\section{INFORMED CONSENT}

Not applicable.

\section{DATA AVAILABILITY STATEMENT}

The datasets used and/or analyzed during the current study are available from the corresponding author on reasonable request.

\section{ORCID}

Muhammad Miftahussurur (iD https://orcid. org/0000-0003-1415-6033

Langgeng Agung Waskito (iD https://orcid. org/0000-0002-9400-1973

Yoshio Yamaoka iD https://orcid.org/0000-0002-1222-5819

\section{REFERENCES}

1. Marshall B, Warren JR. Unidentified curved bacilli in the stomach of patients with gastritis and peptic ulceration. Lancet. 1984;323(8390):1311-1315.

2. Uemura N, Okamoto S, Yamamoto S, et al. Helicobacter pylori infection and the development of gastric cancer. N Engl J Med. 2001;345(11):784-789.

3. Petrosino JF, Highlander S, Luna RA, Gibbs RA, Versalovic J. Metagenomic pyrosequencing and microbial identification. Clin Chem. 2009;55(5):856-866.

4. Nardone G, Compare D. The human gastric microbiota: Is it time to rethink the pathogenesis of stomach diseases? United European Gastroenterol J. 2015;3(3):255-260.

5. Abreu MT, Peek RM. Gastrointestinal malignancy and the microbiome. Gastroenterology. 2014;146(6):1534-1546.e1533.
6. Noto JM, Peek RM Jr. The gastric microbiome, its interaction with Helicobacter pylori, and its potential role in the progression to stomach cancer. PLoS Pathog. 2017;13(10):e1006573-e1006573.

7. Castano-Rodriguez N, Goh KL, Fock KM, Mitchell HM, Kaakoush NO. Dysbiosis of the microbiome in gastric carcinogenesis. Sci Rep. 2017;7(1):15957.

8. The Human Microbiome Project C, Huttenhower C, Gevers D, et al. Structure, function and diversity of the healthy human microbiome. Nature. 2012;486:207.

9. Wu GD, Chen J, Hoffmann C, et al. Linking long-term dietary patterns with gut microbial enterotypes. Science. 2011;334(6052):105-108.

10. Chong CW, Ahmad AF, Lim YAL, et al. Effect of ethnicity and socioeconomic variation to the gut microbiota composition among pre-adolescent in Malaysia. Sci Rep. 2015;5:13338-13338.

11. Liao M, Xie Y, Mao Y, et al. Comparative analyses of fecal microbiota in Chinese isolated Yao population, minority Zhuang and rural Han by 16sRNA sequencing. Sci Rep. 2018;8(1):1142-1142.

12. Deschasaux M, Bouter KE, Prodan A, et al. Depicting the composition of gut microbiota in a population with varied ethnic origins but shared geography. Nat Med. 2018;24(10):1526-1531.

13. Brooks AW, Priya S, Blekhman R, Bordenstein SR. Gut microbiota diversity across ethnicities in the United States. PLoS Biol. 2018;16(12):e2006842.

14. Das A, Pereira V, Saxena S, et al. Gastric microbiome of Indian patients with Helicobacter pylori infection, and their interaction networks. Sci Rep. 2017;7(1):15438-15438.

15. Khosravi Y, Gan HM, Jing P, et al. The gastric microbiome of four Malaysian gastroduodenal disease patients. Arch Gene Genome Res 1(1):1-9.

16. Syam AF, Miftahussurur M, Makmun D, et al. Risk factors and prevalence of Helicobacter pylori in five largest islands of Indonesia: a preliminary study. PLoS ONE. 2015;10(11):e0140186.

17. Klindworth A, Pruesse E, Schweer T, et al. Evaluation of general 16 S ribosomal RNA gene PCR primers for classical and next-generation sequencing-based diversity studies. Nucleic Acids Res. 2013;41(1):e1.

18. Bolger AM, Lohse M, Usadel B. Trimmomatic: a flexible trimmer for Illumina sequence data. Bioinformatics. 2014;30(15):2114-2120.

19. Rognes T, Flouri T, Nichols B, Quince $C$, Mahe F. VSEARCH: a versatile open source tool for metagenomics. PeerJ. 2016;4:e2584.

20. DeSantis TZ, Hugenholtz P, Larsen N, et al. Greengenes, a chimera-checked 16S rRNA gene database and workbench compatible with ARB. Appl Environ Microbiol. 2006;72(7):5069-5072.

21. Katoh K, Standley DM. MAFFT multiple sequence alignment software version 7: improvements in performance and usability. Mol Biol Evol. 2013;30(4):772-780.

22. Katoh K, Misawa K, Kuma K, Miyata T. MAFFT: a novel method for rapid multiple sequence alignment based on fast Fourier transform. Nucleic Acids Res. 2002;30(14):3059-3066.

23. Price MN, Dehal PS, Arkin AP. FastTree 2-approximately maximum-likelihood trees for large alignments. PLoS ONE. 2010;5(3):e9490.

24. Pielou EC. The measurement of diversity in different types of biological collections. J Theor Biol. 1966;13:131-144.

25. Faith DP. Conservation evaluation and phylogenetic diversity. Biol Cons. 1992;61(1):1-10

26. Shannon CE. A mathematical theory of communication. Bell Syst Tech J. 1948;27(3):379-423.

27. Bray JR, Curtis JT. An ordination of the upland forest communities of southern Wisconsin. Ecol Monogr. 1957;27(4):325-349.

28. Oksanen J, Blanchet FG, Kindt R, et al. Vegan: Community Ecology Package. R package version 2.0-2. 2012

29. Segata N, Izard J, Waldron L, et al. Metagenomic biomarker discovery and explanation. Genome Biol. 2011:12(6):R60. 
30. Zakrzewski M, Proietti C, Ellis JJ, et al. Calypso: a user-friendly webserver for mining and visualizing microbiome-environment interactions. Bioinformatics. 2017;33(5):782-783.

31. Dixon M, Genta R, Yardley J, Correa P. Classification and grading of gastritis. The updated Sydney System. International Workshop on the Histopathology of Gastritis, Houston 1994. Am J Surg Pathol. 1996;20(10):1161-1181.

32. Uchida T, Kanada R, Tsukamoto $\mathrm{Y}$, et al. Immunohistochemical diagnosis of the cagA-gene genotype of Helicobacter pylori with anti-East Asian CagA-specific antibody. Cancer Sci. 2007;98(4):521-528.

33. Vilaichone RK, Mahachai V, Shiota S, et al. Extremely high prevalence of Helicobacter pylori infection in Bhutan. World J Gastroenterol. 2013;19(18):2806-2810.

34. Shiota S, Murakami K, Fujioka T, Yamaoka Y. Population-based strategies for Helicobacter pylori-associated disease management: a Japanese perspective. Expert Rev Gastroenterol Hepatol. 2010;4(2):149-156.

35. Shiota S, Cruz M, Abreu JA, et al. Virulence genes of Helicobacter pylori in the Dominican Republic. J Med Microbiol. 2014;63(Pt 9):1189-1196.

36. Nguyen TL, Uchida T, Tsukamoto Y, et al. Helicobacter pylori infection and gastroduodenal diseases in Vietnam: a cross-sectional, hospital-based study. BMC Gastroenterol. 2010;10:114.

37. Nguyen LT, Uchida T, Tsukamoto $\mathrm{Y}$, et al. Clinical relevance of cagPAl intactness in Helicobacter pylori isolates from Vietnam. Eur J Clin Microbiol Infect Dis. 2010;29(6):651-660.

38. Miftahussurur M, Shiota S, Suzuki R, et al. Identification of Helicobacter pylori infection in symptomatic patients in Surabaya, Indonesia, using five diagnostic tests. Epidemiol Infect. 2015;143(5):986-996.

39. Andersson AF, Lindberg $M$, Jakobsson $H$, Backhed F, Nyren $P$, Engstrand L. Comparative analysis of human gut microbiota by barcoded pyrosequencing. PLoS ONE. 2008;3(7):e2836.

40. Li TH, Qin Y, Sham PC, Lau KS, Chu KM, Leung WK. Alterations in gastric microbiota after $\mathrm{H}$. Pylori eradication and in different histological stages of gastric carcinogenesis. Sci Rep. 2017;7:44935.

41. Jeffery IB, Lynch DB, O'Toole PW. Composition and temporal stability of the gut microbiota in older persons. ISME J. 2016;10(1):170-182.

42. O'Toole PW, Jeffery IB. Gut microbiota and aging. Science. 2015;350(6265):1214-1215.
43. Li XX, Wong GL, To KF, et al. Bacterial microbiota profiling in gastritis without Helicobacter pylori infection or non-steroidal anti-inflammatory drug use. PLoS ONE. 2009;4(11):e7985.

44. Miftahussurur M, Syam AF, Nusi IA, et al. Surveillance of Helicobacter pylori antibiotic susceptibility in Indonesia: different resistance types among regions and with novel genetic mutations. PLOS ONE. 2016;11(12):e0166199.

45. Makmun D. Present status of endoscopy, therapeutic endoscopy and the endoscopy training system in Indonesia. Dig Endosc. 2014;26(S2):2-9.

46. Miftahussurur M, Waskito LA, Syam AF, et al. Analysis of risks of gastric cancer by gastric mucosa among Indonesian ethnic groups. PLoS ONE. 2019;14(5):e0216670.

47. Ueki A, Akasaka H, Suzuki D, Ueki K Paludibacter propionicigenes gen. nov., sp. nov., a novel strictly anaerobic, Gram-negative, propionate-producing bacterium isolated from plant residue in irrigated rice-field soil in Japan. Int J Syst Evol Microbiol. 2006;56(Pt 1):39-44.

48. Parsons BN, ljaz UZ, D'Amore R, et al. Comparison of the human gastric microbiota in hypochlorhydric states arising as a result of Helicobacter pylori-induced atrophic gastritis, autoimmune atrophic gastritis and proton pump inhibitor use. PLoS Pasthogens. 2017;13(11):e1006653.

49. Coker OO, Dai Z, Nie Y, et al. Mucosal microbiome dysbiosis in gastric carcinogenesis. Gut. 2018;67(6):1024-1032.

\section{SUPPORTING INFORMATION}

Additional supporting information may be found online in the Supporting Information section.

How to cite this article: Miftahussurur M, Waskito LA, El-Serag HB, et al. Gastric microbiota and Helicobacter pylori in Indonesian population. Helicobacter. 2020;25:e12695. https:// doi.org/10.1111/hel.12695 